\title{
System for simulated in situ measurement of hearing aids
}

\author{
Kaoru Okabe, Hareo Hamada, and Tanetoshi Miura \\ Department of Electrical Communication Engineering, Tokyo Denki University, \\ 2-2, Kanda Nishiki-cho, Chiyoda-ku, Tokyo, 101 Japan
}

(Received 16 September 1983)

\begin{abstract}
A simulated in situ measurement system for hearing aids was developed. The system consists of a head and torso simulator (SAMRAI) with a simplified ear simulator and an equipment for digital signal processing (SPIRIT). SAMRAI was constructed on the basis of the average dimensions of Japanese male adults. The ear simulator, newly developed for practical use, is terminated in a simple resistance of $320 \mathrm{cgs}$ acoustic ohms. The measurement result on SAMRAI with the ear simulator shows good agreement with the real ear data. And several measurement results using this system are discussed. Finally we show a method of measuring vent response using an ear simulator, which has a termination impedance not similar to human eardrum impedance.
\end{abstract}

PACS number: 43. 66. Ts, 43. 66. YW

\section{INTRODUCTION}

In the conventional way of hearing aid measurements, a $2 \mathrm{cc}$ coupler has been used. Although the 2 cc coupler has a simple and stable structure, it is unsatisfactory as a simulator of human ears. Furthermore, in $2 \mathrm{cc}$ coupler measurements, several effects on hearing aids (such as head diffraction, pinna reflection and body baffle effect, which are caused by the presence of the wearer) are not taken into account. As the result, the $2 \mathrm{cc}$ coupler response of a hearing aid differs considerably from actual response on human subjects. Pascoe ${ }^{1)}$ investigated the differences between gains of hearing aids on the $2 \mathrm{cc}$ coupler and those on real ears. He reported that such differences reached about $20 \mathrm{~dB}$ around $3 \mathrm{kHz}$.

For an evaluation of hearing aid performance on a wearer, in situ measurements are required. This method is based on the measurement of sound pressure level (SPL) at the eardrum of a hearing aid wearer. Among the conceptions of in situ measurements, the insertion gain is especially important. It is defined as the difference between SPL with and without a hearing aid, measured at the eardrum of a wearer. The insertion gain, therefore, represents the effective gain of a hearing aid, and it is the objective data to be compared with the hearing level of hearing impaired subjects. So insertion gains provide important guidance for fitting procedures of hearing aids.

For practical reasons, however, it is desirable to substitute a well-defined head and torso simulator (HATS) for human subjects. Burkhard and Sachs ${ }^{2)}$ constructed the HATS based on the average dimensions of male and female American adults. When constructing a HATS, one of the most important and difficult tasks is to develop an ear simulator for HATS. A well-known example is the earlike coupler developed by Zwislocki. ${ }^{3,4)}$ In order to simulate the average human eardrum impedance, he used four elaborate acoustical resonators, which consists of small tubes, cavities and damping materials.

On the other hand, from the technological point of view, another approach is possible; namely, it is to simplify the structure of an ear simulator and utilize it in combination with electrical compensations. From this standpoint, we developed the 
simplified ear simulator and constructed the HATS (SAMRAI) whose dimensions agree with the average Japanese male adult. This paper describes the construction of the simulated in situ measurement system using SAMRAI with the simplified ear simulator and the equipment for digital signal processing (SPIRIT).

\section{IN SITU MEASUREMENT OF HEARING AIDS}

Conceptions of the in situ measurement are illustrated in Fig. 1. The insertion gain is defined by Eq. (1) and it also can be modified to give the difference between the in situ gain and the open ear gain.

$$
\begin{aligned}
\text { Insertion Gain } & =20 \log \frac{p_{\mathrm{h}}}{p_{\mathrm{o}}} \\
& =20 \log \frac{p_{\mathrm{h}} / p_{\mathrm{f}}}{p_{\mathrm{o}} / p_{\mathrm{f}}} \\
& =20 \log \frac{p_{\mathrm{h}}}{p_{\mathrm{f}}}-20 \log \frac{p_{\mathrm{o}}}{p_{\mathrm{f}}} \\
& =\text { In Situ Gain-Open Ear Gain }
\end{aligned}
$$

where $p_{\mathrm{h}}$ denotes eardrum sound pressure with a hearing aid worn, $p_{\mathrm{o}}$ sound pressure under the open ear condition and $p_{\mathrm{f}}$ free field sound pressure at the center position of a wearer's head.

Two conditions that HATSs must satisfy are derived from these definitions. At first, a HATS must

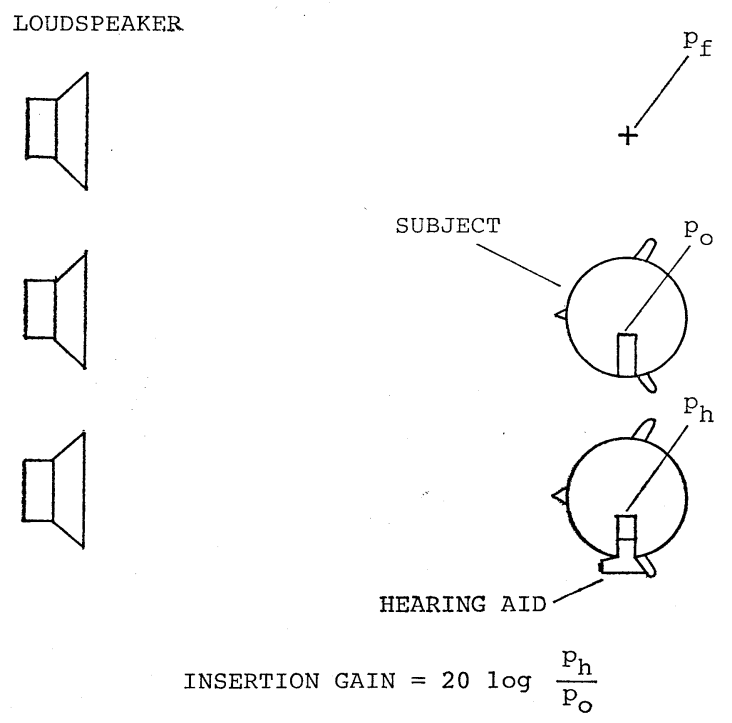

Fig. 1 Definitions of in situ measurement. reproduce the eardrum SPL of human subjects under the open ear condition. Next, the HATS has to simulate the eardrum SPL of subjects with a hearing aid worn.

\section{CONSTRUCTION OF SYSTEM FOR SIMULATED IN SITU MEASUREMENT}

\subsection{Ear Simulator}

In order to reproduce the eardrum SPL under the open ear condition, the transfer function of an ear simulator must be similar to the sound pressure transformation of the average human ear canal.

Next, the ear simulator must have a transfer impedance close to that of real ears, in order to approximate the eardrum SPL of human subjects with an earphone inserted. Because an earphone has normally a very high acoustic impedance, it produces a constant volume velocity. Then, it is necessary to simulate the transfer impedance of real ears, so that the SPL in an ear simulator with an earphone inserted is approximate to the SPL in occluded ears.

However, if an earphone produces a constant volume velocity, the resulting SPL in an ear simulator is completely proportional to the transfer impedance of the ear simulator. Then, even if an ear simulator has a different transfer impedance from that of real ears, the measurement results on it can be compensated uniquely as long as an earphone produces a constant volume velocity.

On the basis of considerations mentioned above, we developed the simplified ear simulator ( $\mathrm{C}$ coupler), which was terminated in a simple resistance element only. The transfer function and transfer impedance of the $\mathrm{C}$ coupler were calculated by using the electrical analog model of the acoustical tube system, shown in Fig. 2. In the case of lossless tubes, the relation between sound pressure and volume velocities in a tube is written by Eq. (2).

$$
\left[\begin{array}{l}
p_{\mathrm{i}} \\
u_{\mathrm{i}}
\end{array}\right]=\left[\begin{array}{cc}
\cos k l & j Z_{0} \sin k l \\
j \frac{1}{Z_{0}} \sin k l & \cos k l
\end{array}\right]\left[\begin{array}{l}
p_{\mathrm{t}} \\
u_{\mathrm{t}}
\end{array}\right]
$$

Then, the transfer function of the $\mathrm{C}$ coupler can be calculated from Eq. (3), which is derived from Eq. (2).

$$
H=\frac{p_{\mathrm{t}}}{p_{\mathrm{i}}}=\frac{1}{\cos k l+j \frac{Z_{\mathrm{o}}}{Z_{\mathrm{t}}} \sin k l} .
$$




\section{K. OKABE et al.: SYSTEM FOR SIMULATED IN SITU MEASUREMENT}
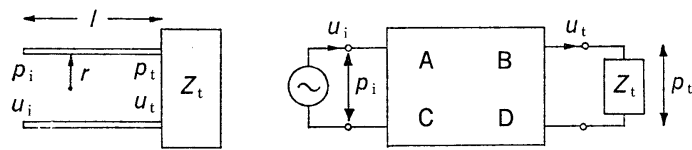

$\left[\begin{array}{l}p_{\mathrm{i}} \\ u_{\mathrm{i}}\end{array}\right]=\left[\begin{array}{lc}\cos k l & j Z_{\mathrm{o}} \sin k l \\ j \frac{1}{Z_{\mathrm{o}}} \sin k l & \cos k l\end{array}\right]\left[\begin{array}{l}p_{\mathrm{t}} \\ u_{\mathrm{t}}\end{array}\right]$

where

$$
\begin{aligned}
j & =\sqrt{-1}, \quad Z_{\mathrm{o}}=\frac{\rho c}{\pi r^{2}}, \quad k=\frac{2 \pi f}{c} \\
p_{\mathrm{i}}, p_{\mathrm{t}}: & \text { sound pressure } \\
u_{\mathrm{i}}, u_{\mathrm{t}}: & \text { volume velocity } \\
l: & \text { length of acoustic tube } \\
r: & \text { radius of acoustic tube } \\
c: & \text { sound velocity } \\
\rho: & \text { density of air } \\
f: & \text { frequency }
\end{aligned}
$$

Fig. 2 Acoustical tube and its electrical representation.

By substituting the resistance of $320 \mathrm{cgs}$ acoustic ohms for $Z_{\mathrm{t}}, 2.15 \mathrm{~cm}$ for $l$ and $0.375 \mathrm{~cm}$ for $r$, we obtain the transfer function of the $\mathrm{C}$ coupler, shown in Fig. 3. In the same figure, the broken line represents the transfer function of a real ear model. This model is defined as the theoretical model having a cylindrical ear canal and the eardrum impedance proposed by Shaw. ${ }^{5}$ )

The transfer impedance of a lossless tube $Z_{\mathrm{tr}}$ can be calculated from Eq. (4).

$$
Z_{\mathrm{tr}}=\frac{p_{\mathrm{t}}}{u_{\mathrm{i}}}=\frac{1}{\frac{1}{Z_{\mathrm{t}}} \cos k l+j \frac{1}{Z_{\mathrm{o}}} \sin k l} .
$$

In the case that an ear canal is occluded by earphones, $l=1.27 \mathrm{~cm}$ for the average distance between the eardrum and the earmold tip. Figure 4 shows the computation results of the $\mathrm{C}$ coupler and the real ear model. The ordinate represents the magnitude of the transfer impedance in $\mathrm{dB}$ referred to $1 \mathrm{cgs}$ acoustic ohm. The discrepancy between two curves arises below $0.7 \mathrm{kHz}$ and it increases as the frequency decreases. The discrepancy is due to differences of the termination impedance between the $\mathrm{C}$ coupler and the real ear model. The termination impedance of the $\mathrm{C}$ coupler is only resistive, but on the other hand the human eardrum impedance consists of both the compliance and the resistance in this frequency range. Because of differences in the

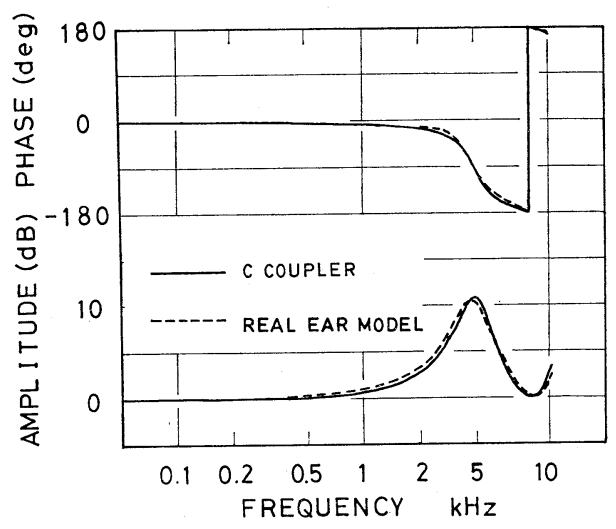

Fig. 3 Calculated transfer function of the $\mathrm{C}$ coupler and the real ear model. Solid line represents the result of the $\mathrm{C}$ coupler and the broken line the real ear model.

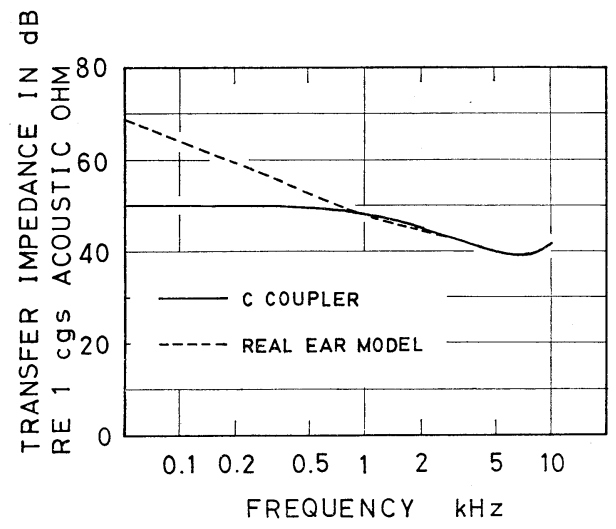

Fig. 4 Calculated transfer impedance of the $\mathrm{C}$ coupler and the real ear model.

The solid line represents the result of the $\mathrm{C}$ coupler and the broken line the real ear model. Ordinate shows the impedance magnitude in $\mathrm{dB}$ referred to $1 \mathrm{cgs}$ acoustic ohm.

transfer impedance, the measured earphone response on the $\mathrm{C}$ coupler differs from that obtained on real ears. However, the measured response can be compensated uniquely, as mentioned above.

Figure 5 shows the structure of the $\mathrm{C}$ coupler. The termination impedance of $320 \mathrm{cgs}$ acoustic ohms is realized by an acoustical tube having a diameter of $4 \mathrm{~mm}$ and a length of about $5 \mathrm{~m}$. The end of the tube is closed and rounded. 


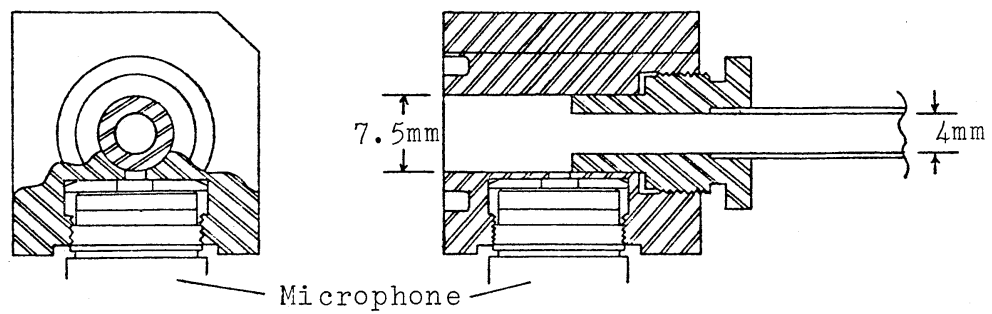

Fig. 5 Structure of the $\mathrm{C}$ coupler.

Termination impedance of the coupler is an acoustical tube with $4 \mathrm{~mm}$ in diameter and about $5 \mathrm{~m}$ in length. The end of the tube is closed and rounded.

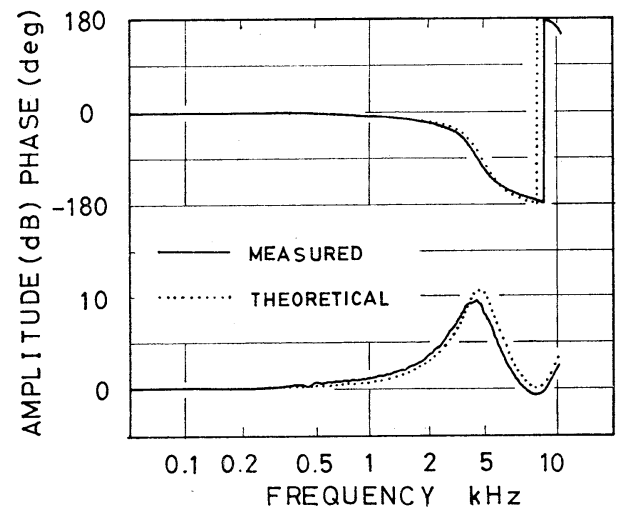

Fig. 6 Measured transfer function of the C coupler.

Dotted line shows the theoretical curve.

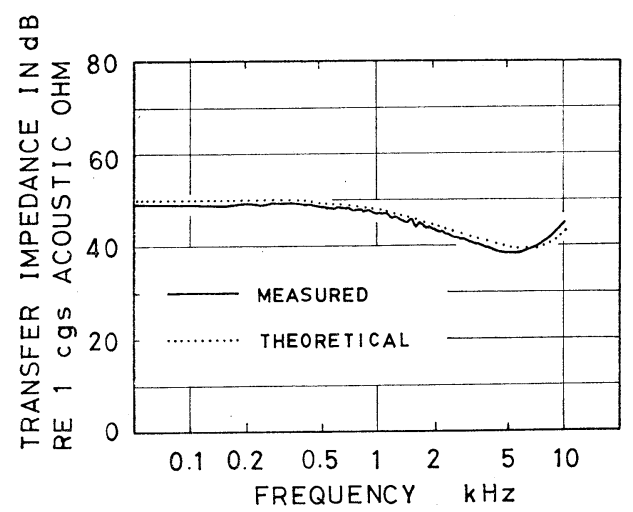

Fig. 7 Transfer impedance of the C coupler. Data were calculated on the basis of its measured input impedance.

The measured transfer function of the $\mathrm{C}$ coupler is shown in Fig. 6 and is compared with the calculated curve.
In order to confirm the transfer impedance of the $\mathrm{C}$ coupler, we measured its input impedance $Z_{\mathrm{in}}$ and estimated its transfer impedance $Z_{\mathrm{tr}}$ from the measured input impedance, using Eq. (5).

$$
Z_{\mathrm{tr}}=\left[j \frac{1}{Z_{\mathrm{o}}} \sin k l+\frac{\cos k l-\frac{Z_{\text {in }}}{Z_{\mathrm{o}}} \sin k l}{Z_{\text {in }} \cos k l-j Z_{\mathrm{o}} \sin k l} \cos k l\right]^{-1} .
$$

The input impedance of the $\mathrm{C}$ coupler was measured by using an acoustical tube. ${ }^{6)}$ Figure 7 shows the result, where the broken line represents the theoretical curve.

The measured data agree with theoretical values within the order of impedance measurement errors.

\subsection{Head and Torso Simulator (SAMRAI)}

SAMRAI was constructed on the basis of the average dimensions of Japanese male adults. The acoustical characteristics of SAMRAI with a C coupler are shown in the following two figures. In Fig. 8, the measured transfer function of SAMRAI's ear canal (the same data shown in Fig. 6) is compared with the real ear data by Yamaguchi and Sushi, ${ }^{7)}$ and Shaw. ${ }^{8)}$

Figure 9 represents the open ear gain of SAMRAI and the real ear data. ${ }^{7,8)}$ From comparisons in this figure, it can be confirmed that SAMRAI properly simulates the eardrum SPL under open ear conditions.

In the following stage of our study, we measured the insertion gain of behind-the-ear type hearing aids on SAMRAI and compared the results with the data on real ears. Figure 10 shows the measured results. The real ear data were measured on three male subjects with normal hearing. We used a probe tube microphone to measure SPL in the ear canals and set the probe orifice at a point of $5 \mathrm{~mm}$ away 


\section{K. OKABE et al:: SYSTEM FOR SIMULATED IN SITU MEASUREMENT}

from the earmold tips.

According to Burkhard and Sachs, ${ }^{9}$ this position ensures plane wave propagation in the ear canal.

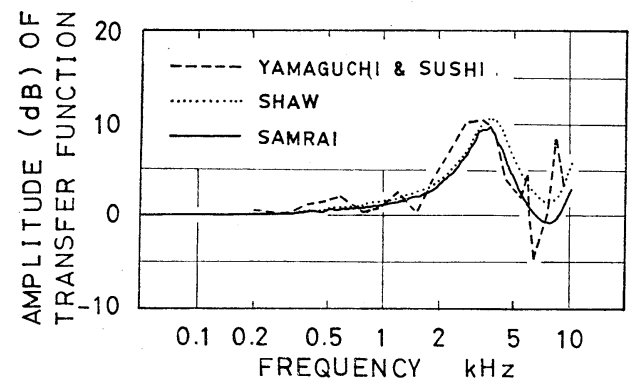

Fig. 8 Measured transfer function of SAMRAI's ear canal and real ear data by Yamaguchi and Sushi ${ }^{7)}$ and by Shaw. ${ }^{8)}$

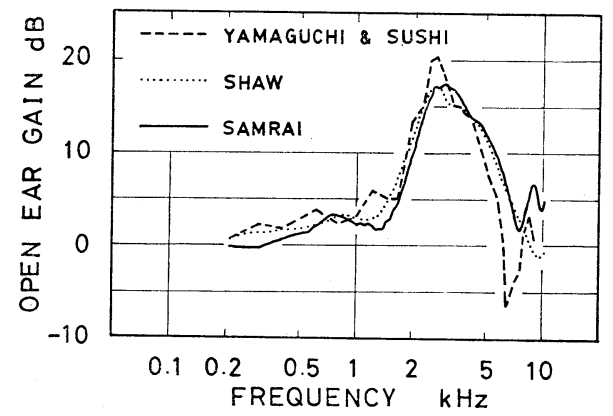

Fig. 9 SAMRAI open ear gain and real ear data by Yamaguchi and Sushi ${ }^{7)}$ and by Shaw. ${ }^{8)}$

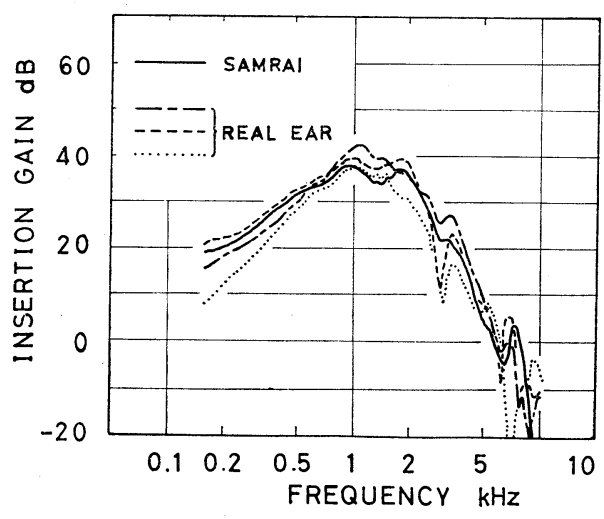

Fig. 10 Simulated insertion gain of a behind-the-ear hearing aid on SAMRAI and measured insertion gains on three real ears.
We measured the SPL with and without a hearing aid at this position. Because of the fixed position of the probe orifice in the ear canals, the differences between the measured SPL with and without a hearing aid provide the insertion gain. In this figure, variations in the individual curves become large in the high frequency regions, and the simulated insertion gain on SAMRAI agrees qualitatively with the real ear data.

No compensations for the transfer impedance difference were made to the result on SAMRAI. From the theoretical point of view, the measured result on SAMRAI must differ from the real ear data below $0.7 \mathrm{kHz}$, because the $\mathrm{C}$ coupler has small values of the transfer impedance relative to real ears in this frequency region. On the contrary, there appear to be no obvious discrepancies among the measured responses. This may be due to natural leakages around the earmold of each subject. Then, it is necessary to compensate the measured result on SAMRAI for the simulation of complete coupling between the ear canal and the earmold.

\subsection{Construction of Measurement System}

Characteristics of the electro-acoustic transducer system can be specified from its impulse response, if it is linear and time-invariant. This method is not applicable to a non-linear system such as a hearing aid with auto gain control (AGC) in operation. However, if a hearing aid works in its linear region, we can measure the characteristics of the aid from its impulse response.

We constructed a simulated in situ measurement system which consists of SAMRAI with a $\mathrm{C}$ coupler and equipment for digital signal processing, named SPIRIT (System for Processing of Impulse Response based on In situ measurement Technique). Figure 11 represents a block diagram of the whole system. It is a digitalized system, which measures the impulse response of a hearing aid and computes its frequency response. SPIRIT generates a $4 \mu \mathrm{s}$ impulse. The impulse is fed to a loudspeaker, and the impulse response is picked up by a condenser microphone at the "eardrum" position of SAMRAI. The impulse response is sampled with a $50 \mathrm{kHz}$ sampling frequency and quantized by a 12 bit A/D converter and stored in the memory. In order to increase $\mathrm{SN}$ ratio, one can select the number of times in averaging among 128, 256, 512, 1,024, and 2,048. The averaged impulse response is transformed into the 


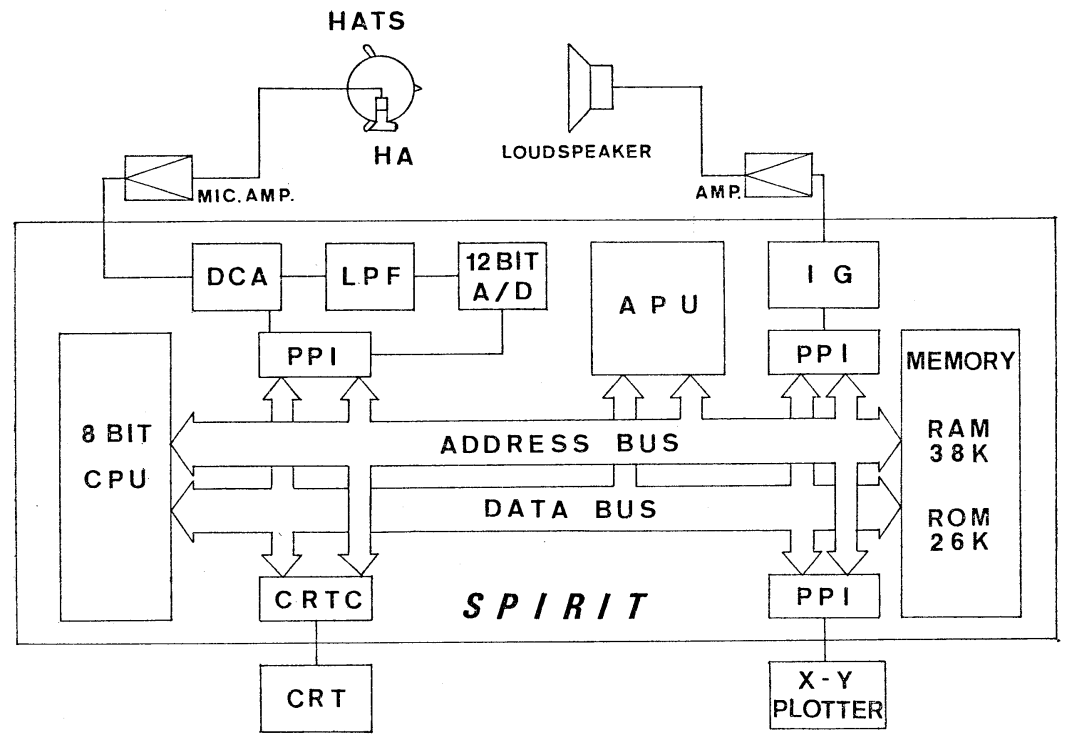

Fig. 11 Block diagram of the simulated in situ measurement system.

APU: arithmetic processing unit

DCA: digital controled att.

A/D: analog/digital converter

IG: impulse generator
HA: hearing aid

LPF: low pass filter

PPI: programmable peripheral interface

$\mathrm{S} / \mathrm{H}$ : sample/hold frequency domain data using a FFT (fast Fourier transform) algorithm. SPIRIT can store two sorts of frequency domain data at a time and compute the insertion gain or the vent response from the stored data. We developed an algorithm to transform the vent response on the $\mathrm{C}$ coupler into the response on the average real ear (see Appendix).

\section{MEASUREMENT RESULTS}

Examples of measured results using SPIRIT and SAMRAI are shown in this chapter. Figure 12 (a) shows the impulse response measured on SAMRAI under the open ear condition. And Fig. 12 (b) shows the response of a behind-the-ear (BTE) type hearing aid on SAMRAI. The amplitude of impulse responses is arbitrary.

Figure 13 shows the simulated insertion gain of the aid, calculated from impulse responses in Fig. 12. For a comparison, the $2 \mathrm{cc}$ coupler gain of the aid is shown in the same figure. The simulated insertion gain is lower than the $2 \mathrm{cc}$ coupler gain at high frequencies, and the difference between the two gains reaches about $20 \mathrm{~dB}$ around $3 \mathrm{kHz}$. This shows good agreement with the data by Pascoe. ${ }^{1)}$ In this measurement, we used an earmold simulator for
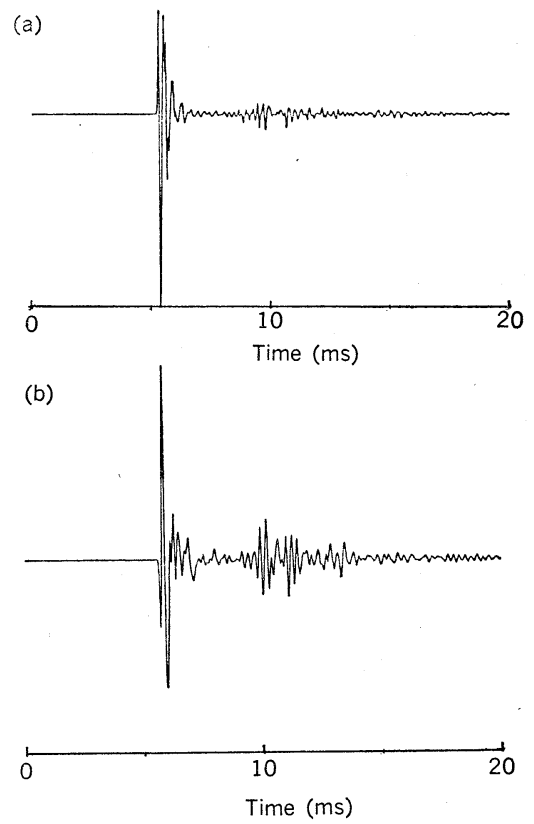

Fig. 12 Measured impulse response on SAMRAI under the open ear condition (a) and the response on SAMRAI with a hearing aid worn (b). 


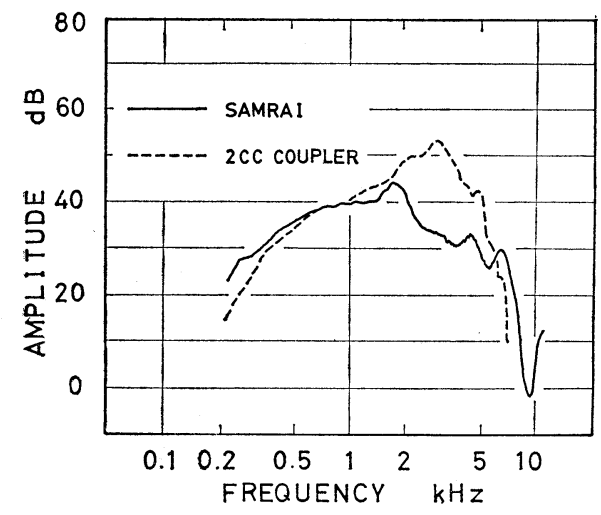

Fig. 13 Comparison of simulated insertion gain on SAMRAI and $2 \mathrm{cc}$ coupler gain.
SAMRAI, which has typical earmold dimensions; the inner diameter of the earmold is $2 \mathrm{~mm}$ and the sound tube length about $4 \mathrm{~cm}$.

Figures 14 and 15 represent the differences between the simulated insertion gains and the $2 \mathrm{cc}$ coupler gains. The data in Fig. 14 were measured with three BTE type hearing aids, and Fig. 15 with four inthe-ear (ITE) type aids. The letters A, B, C, and D indicate the location of a hearing aid microphone. In these measurements, the earmold simulator for SAMRAI has same dimensions in its length and diameter that are used in the $2 \mathrm{cc}$ coupler measurements. In the frequency range from $2 \mathrm{kHz}$ to 5 $\mathrm{kHz}$, all the insertion gain values fall below the $2 \mathrm{cc}$ coupler gain. The gain differences of the BTE aids have a different pattern from those of the ITE aids. The variations in Fig. 15 indicate that the

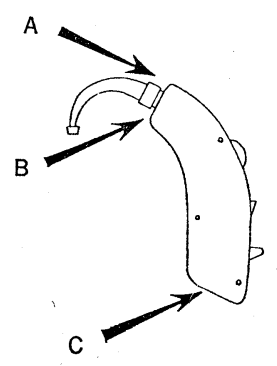

Location of hearing aid microphone

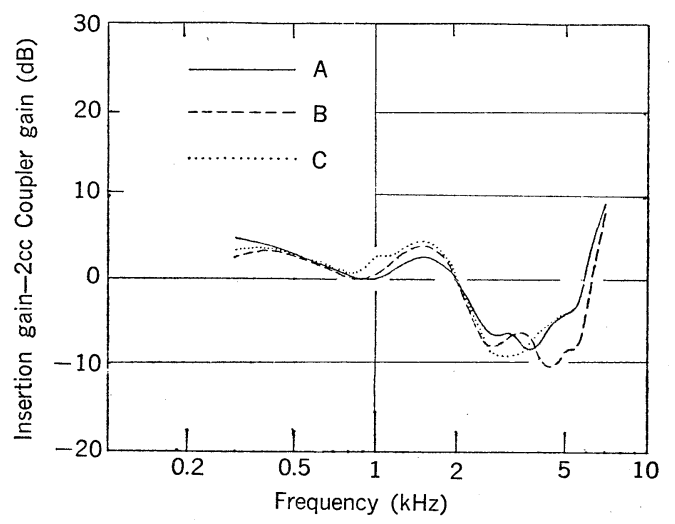

Fig. 14 Differences between simulated insertion gain on SAMRAI and $2 \mathrm{cc}$ coupler gain (behind-the-ear type).

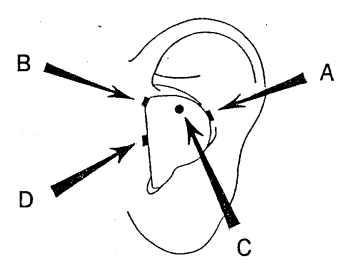

Location of hearing aid microphone

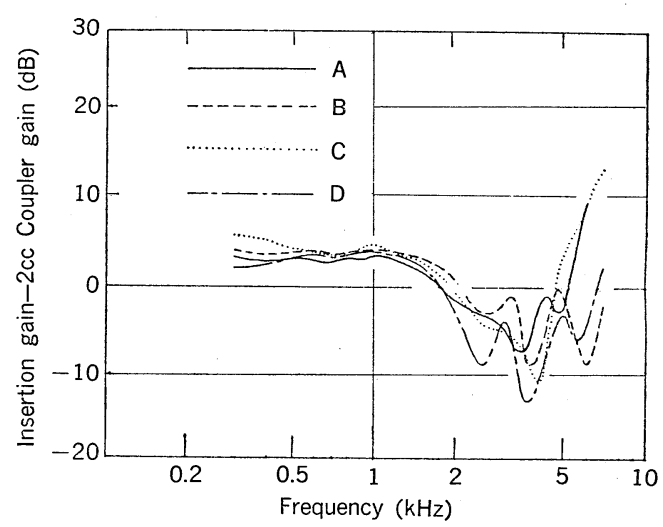

Fig. 15 Differences between simulated insertion gain on SAMRAI and $2 \mathrm{cc}$ coupler gain (in-the-ear type). 


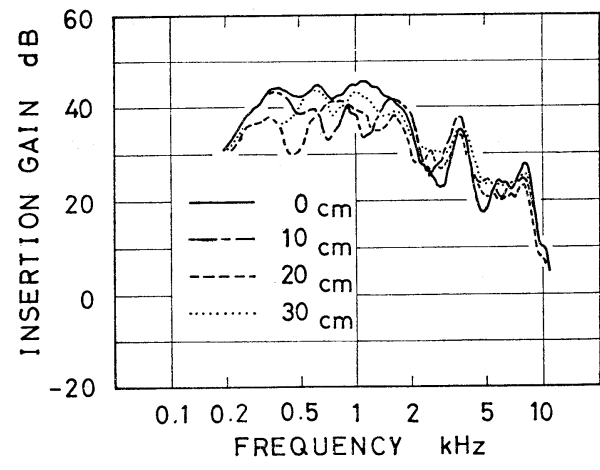

Fig. 16 Variations of simulated insertion gain of a box type aid when the aid was moved away from body surface of SAMRAI by $10 \mathrm{~cm}$ steps.

insertion gain of ITE aids varies considerably according to the microphone location.

Figure 16 shows the variations of the simulated insertion gain of a box type hearing aid, which were observed when the aid was moved away from the body surface of SAMRAI by 10,20 , and $30 \mathrm{~cm}$ steps. The insertion gain at the low frequencies changes considerably because of the variations of the body baffle effect on the hearing aid. Corresponding to the time delay of the reflected wave from the body surface, the dip in the low frequencies varies its position.

\section{CONCLUSION}

The simplified ear simulator for hearing aid measurements was evaluated theoretically and experimentally. Using the ear simulator and the HATS (SAMRAI) in combination with proper compensations, one can obtain measurement results similar to results on human subjects.
Equipment for digital signal processing (SPIRIT) was also developed, and the total system for the simulated in situ measurement was constructed. By using this system, it was shown that insertion gains of hearing aids, vent responses and evaluations of some acoustical effects on aids can be measured.

It is also possible to apply SAMRAI to some additional uses, such as research for subjective judgment of hearing aid performance or determining binaural effects when using two hearing aids. For the development of better hearing aid fitting procedures, the simplified ear simulator and SAMRAI can be utilized for objective and subjective evaluations of hearing aids.

\section{ACKNOWLEDGMENTS}

The authors are indebted to Dr. Kenji Nakamura for his helpful suggestions. The authors also wish to thank Mr. Yasuo Ogura and Mr. Kenichi Saito for their stimulating discussions.

\section{APPENDIX}

The vent response is defined as the difference between earphone response with and without a vent in an earmold. Vent responses depend on the measuring coupler, because the responses are sensitive to the input impedance of the measuring coupler. Figure 17 illustrates the vented and unvented earmolds and their electrical analogs.

As mentioned before, the termination impedance of a $\mathrm{C}$ coupler differs from the human eardrum impedance, therefore, the input impedance of a $\mathbf{C}$ coupler is not same as that of the real ear. So the vent response on a $\mathrm{C}$ coupler is different from that obtained with the real ear.

In this appendix, we show a method to predict vent responses on the real ear using the coupler, which has a different input impedance from that of
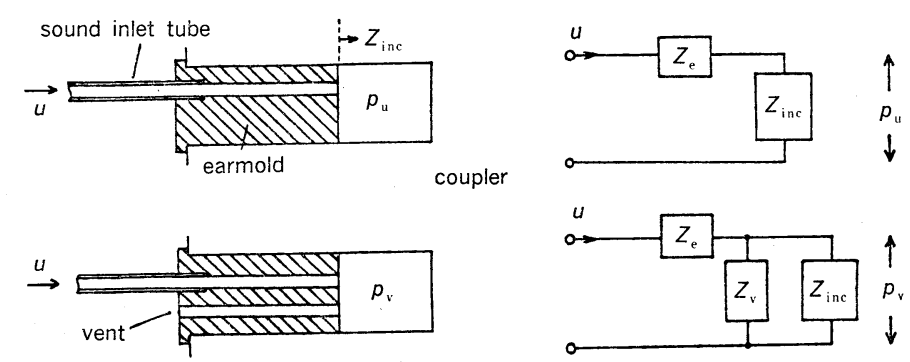

Fig. 17 Earmolds and coupler and their electrical analog models. 


\section{K. OKABE et al.: SYSTEM FOR SIMULATED IN SITU MEASUREMENT}

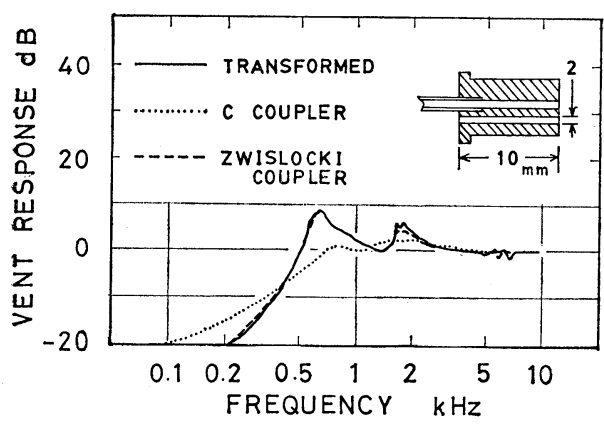

Fig. 18 Vent response on the $\mathrm{C}$ coupler and its transformed result.

Broken line represents the measured response on the Zwislocki type coupler.

the real ear. Taking account of the fact that an earphone produces a constant volume velocity, the vent response on a coupler $H_{\mathrm{c}}$ is expressed by Eq. (A1). ${ }^{10)}$

$$
H_{\mathrm{c}}=\frac{p_{\mathrm{v}}}{p_{\mathrm{u}}}=\frac{Z_{\mathrm{v}}}{Z_{\mathrm{v}}+Z_{\text {inc }}}
$$

where $Z_{v}$ denotes the impedance of a vent opening and $Z_{\text {ine }}$ the input impedance of the coupler.

Similarly, the vent response on the real ear $H_{\mathrm{r}}$ can be written by Eq. (A2).

$$
H_{\mathrm{r}}=\frac{Z_{\mathrm{v}}}{Z_{\mathrm{v}}+Z_{\mathrm{inr}}}
$$

where $Z_{\text {inr }}$ denotes the input impedance of the real ear.

By substituting Eq. (A1) into Eq. (A2), we get Eq. (A3).

$$
H_{\mathrm{r}}=\frac{Z_{\text {inc }} H_{\mathrm{c}}}{Z_{\text {inc }} H_{\mathrm{c}}+\left(1-H_{\mathrm{c}}\right) Z_{\text {inr }}} .
$$

Equation (A3) provides the transformation from the vent response on a coupler to the response on the real ear.

In order to confirm the method experimentally, we measured the vent response on the $\mathrm{C}$ coupler, and transformed it to the response on the Zwislocki type coupler using Eq. (A3). The input impedance of the $\mathrm{C}$ coupler and Zwislocki type coupler were measured by using an acoustical tube. ${ }^{6)}$

Figure 18 shows the results. The dotted line represents the vent response on the $\mathrm{C}$ coupler and the solid line the transformed response using Eq. (A3). The broken line represents the measured vent response on the Zwislocki type coupler. The predicted result shows excellent agreement with the measured response on the Zwislocki type coupler.

\section{REFERENCES}

1) D. P. Pascoe, "Frequency responses of hearing aids and their effects on the speech perception of hearing impaired subjects," Ann. Otol. Rhinol. Laryngol. 84, 1-44 (1975).

2) M. D. Burkhard and R. M. Sachs, "Anthropometric manikin for acoustical research," J. Acoust. Soc. Am. 58, 214-222 (1975).

3) J. J. Zwislocki, "An acoustic coupler for earphone calibration," Special Rep. LSC-S-7 (Syracuse University, Syracuse, New York, 1970).

4) J. J. Zwislocki, "An ear-like coupler for earphone calibration," Special Rep. LSC-S-9 (Syracuse University, Syracuse, New York, 1971).

5) E. A. G. Shaw, "The external ear," Handbook of Sensory Physiology, ed. by W. D. Keidel and W. D. Neff (Springer-Verlag, Berlin, 1974), Vol. V/1, Chap. 14, p. 472.

6) H. Hamada, K. Saito, Y. Ogura, and T. Miura, "Acoustic impedance measurement of human ear," Proc. Tenth Int. Congr. Acoust. (Sydney), M-4.1 (1980).

7) Z. Yamaguchi and N. Sushi, "Real ear response of receivers," J. Acoust. Soc. Jpn. 12, 8-23 (1956) (in Japanese).

8) E. A. G. Shaw, "Transformation of sound pressure level from the free field to the eardrum in the horizontal plane," J. Acoust. Soc. Am. 56, 18481861 (1974).

9) M. D. Burkhard and R. M. Sachs, "Sound pressure in insert earphone coupler and real ears," J. Speech Hear. Res. 20, 799-807 (1977).

10) S. F. Lybarger, "Earmold venting as an acoustical factor," Acoustical Factors Affecting Hearing Aid Performance, ed. by G. A. Studebaker and I. Hochberg (University Park Press, Baltimore, 1980), Chap. 10, p. 199. 